Stephan Kraft

\title{
Wider den „Hauffen junger Schnautzhahnen“
}

\author{
Grimmelshausens Versuch, die Erinnerung an den \\ Dreißigjährigen Krieg in der Gegenwart festzuhalten
}

DOI 10.1515/iasl-2017-0005

\begin{abstract}
This paper retraces the specific conception of the 'present' as manifested in Satyrischer Pilgram, Grimmelshausen's first published work. In the face of progressively vanishing consciousness of the past terrors of the Thirty Years' War, the Pilgram devises a program for bringing the experience of a whole generation to the present, thereby saving it for the future. Instead of delivering a general reflection on the nature of war, it suggests to narrate individually experienced "particularities." It is crucial that all of these experiences are negative, not meant to prompt imitation, but instead rather to build and keep up a stronghold against attitudes and actions of the past.
\end{abstract}

\section{Grimmelshausens Romane zwischen Exempel und Ereignis}

Schon seitdem die simplicianischen Schriften Hans Jacob Christoffel von Grimmelshausens im 19. Jahrhundert in das Blickfeld der germanistischen Forschung geraten sind, wird mit wechselnden Akzentsetzungen darüber diskutiert, ob es sich hier eher um Texte handelt, die ihren Schwerpunkt im zeitgenössisch Ereignishaften haben oder ob hier nicht doch das zeitenthobene Exemplarische im Zentrum stehe. Nachdem in der Frühzeit der Beschäftigung zumeist eine vermeintliche Unmittelbarkeit der Erlebnisschilderung eines unverbildeten Bauernpoeten postuliert worden war, dominierten in weiten Teilen des 20. Jahrhunderts Betrachtungsweisen, nach denen in den simplicianischen Romanen exemplarisch die Verfallenheit des Irdischen und die Möglichkeit eines Weges zum Seelenheil modelliert würden, sowie Untersuchungen, die die Texte auf ihre Quellen hin

Kontaktdaten: Prof. Dr. Stephan Kraft, Universität Würzburg, Institut für deutsche Philologie, Am Hubland, D-97074 Würzburg, E-Mail: stephan.kraft@uni-wuerzburg.de 
untersuchten oder sie in literarische Traditionslinien, wie etwa die des Pikarischen, einrückten.

Erst zuletzt wurde wieder verstärkt über ein Heraustreten der Romane aus den üblichen Diskurszusammenhängen der barocken Tradition nachgedacht. ${ }^{1}$ Wenn der Dreißigjährige Krieg bei Grimmelshausen als ein im Wortsinne unerhörter Schrecken gefasst wird, so schwächt sich damit notwendig auch die Möglichkeit der Einrückung der Geschehnisse in die traditionellen Exempelketten. Entgegen dem im 17. Jahrhundert noch topischen „Es gibt nichts Neues unter der Sonne“ (Koh 1,9) von König Salomo erscheint hier ein wirklich ungesehener und damit diskursiv nicht mehr so einfach zu bändigender Horror. ${ }^{2}$

Diese Inkommensurabilität manifestiert sich in Grimmelshausens Simplicissimus Teutsch und seinen Nachfolgetexten vor allem in Form von Bruchstellen zwischen dem fortgesetzten Bemühen um die Darstellung der Welt als einer am Ende doch sinnvollen und dem zugleich als notwendig präsentierten Scheitern dieser Anstrengungen. Nachvollziehen lässt sich dies beispielsweise an der Darstellung des Überfalls der Reiter auf den Bauernhof im Spessart gleich zu Beginn des ersten Buches. Einer intensiven Beschwörung der Schrecken des erlebenden kindlichen Ich steht hier eine Einordnung gegenüber, die das erzählende Ich in Form des zurückschauenden älteren Eremiten Simplicissimus vornimmt: ${ }^{3}$

So erfordert jedoch die Folge meiner Histori / daß ich der lieben posterität hinterlasse / was vor Grausamkeiten in diesem unserm Teutschen Krieg hin und wieder verübet worden / zumalen mit meinem eigenen Exempel zu bezeugen / daß alle solche Ubel von der Güte deß Allerhöchsten / zu unserm Nutz / offt notwendig haben verhängt werden müssen: Dann lieber Leser / wer hätte mir gesagt / daß ein GOtt im Himmel wäre / wann keine Krieger

1 Vgl. dazu u.a. Andreas Merzhäuser: Satyrische Selbstbehauptung. Innovation und Tradition in Grimmelshausens „Abentheurlichem Simplicissimus Teutsch“. Göttingen: Wallstein 2002. Etwas zurückhaltender, prinzipiell aber ebenfalls an Fragen der Modernität bei Grimmelshausen interessiert ist etwa Italo Michele Battafarano: Simplicana Bellica. Grimmelshausens Kriegsdarstellung und ihre Rezeption 1667-2006. Bern u.a.: Peter Lang 2011.

2 Dies steht auch mit der zuletzt von historiografischer Seite erneut vertieften These im Zusammenhang, dass das Phänomen der Verzeitlichung, das ab dem späteren 18. Jahrhundert das moderne Geschichtsverständnis dominieren wird, in der Inkommensurabilität dieser spezifischen frühneuzeitlichen Krisenerfahrung einen seiner Ursprünge gehabt habe. Vgl. etwa Hans-Joachim von Kondratowitz: Generationenbewusstsein. In: Friedrich Jäger (Hg.): Enzyklopädie der Neuzeit. Stuttgart: Metzler 2005-2012. Bd. 4. Stuttgart: Metzler 2006, Sp. 448-450. Allgemeiner situiert den Ursprung eines modernen Gegenwarts- und damit Geschichtskonzepts in den ubiquitären Krisenerfahrungen des 17. Jahrhunderts Achim Landwehr: Geburt der Gegenwart. Eine Geschichte der Zeit im 17. Jahrhundert. Frankfurt/M.: Fischer 2014, insbesondere S. 196-203: „Warum das 17. Jahrhundert?“

3 Vgl. ausführlicher zu diesem „Multiperspektivismus der Ich-Form“ auch Battafarano: Simpliciana Bellica (Anm. 1), S. 45-57. 
meines Knans Hauß zernichtet / und mich durch solche Fahung unter die Leut gezwungen hätten [...]? [...] Und wiewol er tausenderley Weg hierzu hatte / wolte er sich doch ohn zweiffel nur deß jenigen bedienen / in welchem mein Knan und Meüder / andern zum Exempel / wegen ihrer liederlichen Aufferziehung gestrafft würden. ${ }^{4}$

Auch solche Gräueltaten, wie die auf dem Bauernhof geschehenen, hätten demnach ihren Ort in Gottes Heilsplan, denn zum einen stellten sie eine gerechte Strafe für seine (Zieh-)Eltern dar, die ihn bis dahin so gottesfern aufgezogen hätten, und zum anderen habe er selbst erst durch seine Vertreibung vom elterlichen Hof den Weg zum Christentum und damit zum Heil gefunden. Dass hier aber keinesfalls nur die vermeintlich schuldigen Eltern dem Strafgericht ausgeliefert waren, sondern dass sich auch Kinder unter den Opfern fanden und junge Mägde wahllos vergewaltigt wurden, wird vom älteren Simplicissimus in seinen Erklärungsbemühungen geflissentlich ausgeblendet. Die Frage stellt sich, wessen Reaktion am Ende die angemessenere ist - die des vor den Gräueln fassungslos dastehenden kindlichen Ich oder die des erzählenden Ich, das uns die Zusammenhänge von vermeintlicher Ursache und gerechter Folge explizieren zu können meint. $^{5}$

Es entsteht somit, wie bereits angedeutet, ein doppelpoliges Kraftfeld zwischen dem fortgeführten Versuch einer Verarbeitung des Schreckens durch eine Integration in die bereitliegenden Diskurse und dessen drohendem Scheitern am Material selbst. In gewisser Weise analog zur Französischen Revolution etwa anderthalb Jahrhunderte später scheint die Kriegserfahrung hier dazu geeignet, die Geschichte in ein Davor und in ein Danach zu teilen - allerdings mit dem gewichtigen Unterschied, dass im 17. Jahrhundert jenseits des apokalyptischen Denkens eben noch kein Diskurs zur Verfügung stand, mit dessen Hilfe die Neuheit dieser Katastrophe direkt hätte artikuliert werden und in den jemand wie Grimmelshausen dann einfach hätte einstimmen können.

Apokalyptische Geschichtskonzepte werden von Grimmelshausen zwar immer einmal wieder aufgerufen, wie es etwa gleich im ersten Satz des Simplicissimus Teutsch selbst geschieht, in dem es von der „dieser unserer Zeit“ heißt, dass man glaube, „daß es die letzte seye“. ${ }^{6}$ Allerdings dominieren bei ihm, wie sich im

4 Hans Jacob Christoffel von Grimmelshausen: Der abentheurliche Simplicissimus Teutsch. In: H.J.C.G.: Werke in drei Bänden. Hg. von Dieter Breuer. Frankfurt/M.: Deutscher Klassiker Verlag 1989ff. Band I.1. Frankfurt/M.: Deutscher Klassiker Verlag 1989, S. 27.

5 Vgl. zu dieser Argumentation auch Andreas Merzhäuser: Über die Schwelle geführt. Anmerkungen zur Gewaltdarstellung in Grimmelshausens „Simplicissimus“. In: Markus Meumann/Dirk Niefanger (Hg.): Ein Schauplatz herber Angst. Wahrnehmung und Darstellung von Gewalt im 17. Jahrhundert. Göttingen: Wallstein 1997, S. 65-82.

6 Grimmelshausen: Simplicissimus Teutsch (Anm. 4), S. 17. 
Verlauf der Argumentation zeigen wird, am Ende doch sehr praktische Reflexionen zum Umgang mit einer diesseitigen Welt, die noch keineswegs endgültig verloren gegeben worden ist.

\section{Der Satyrische Pilgram und seine „Vorred“ - ein Autor präsentiert sich und sein Programm}

Diskutiert wurde die hier aufgegriffene Grundfrage bislang zumeist anhand der Romane Grimmelshausens und vor allem natürlich anhand des Simplicissimus Teutsch selbst. An dieser Stelle soll allerdings eine andere Schrift des Verfassers ins Zentrum gerückt werden, nämlich sein 1666/1667 veröffentlichtes Erstlingswerk Satyrischer Pilgram, in dem Grimmelshausen sein Autorkonzept präsentiert sowie sein im Folgenden umgesetztes Schreibprogramm begründet. ${ }^{7}$ Es wird sich zudem zeigen, dass sich bereits in diesem frühen Text einige Schlüsselstellen für die Themen der Gegenwärtigkeit und der damit zusammenhängenden Verzeitlichung finden, die zugleich den historischen Horizont nochmals ausweiten. Neben der besonderen temporalen Situierung des Dargestellten erhält dabei auch die Seite der Darstellung selbst eine Zeitsignatur. Warum - so steht als eine Frage im Raum, die im Satyrischen Pilgram auch explizit reflektiert wird - dauerte es nach dem Ende des Krieges nochmals rund 20 Jahre, bis Grimmelshausen in dieser Sache öffentlich das Wort ergreift? ${ }^{8}$

7 Hans Jacob Christoffel von Grimmelshausen: Satyrischer Pilgram. Hg. von Wolfgang Bender. Tübingen: Niemeyer 1970. Die Zahl der Studien, die sich auf diesen Text Grimmelshausens konzentrieren, ist überschaubar. Vgl. etwa Wilhelm Kühlmann: „Syllogismus practicus“ - Antithese und Dialektik in Grimmelshausens „Satyrischem Pilgram“. In: Simpliciana XIII (1991), S. 391-405; Rainer Hillenbrand: Zu Grimmelshausens „Satyrischem Pilgram“. In: Archiv für das Studium der neueren Sprachen und Literaturen 148 (1996), S. 326-330. Eine zentrale Rolle nimmt dieser Traktat auch bei Merzhäuser ein. Vgl. Merzhäuser: Satyrische Selbstbehauptung (Anm. 1), unter anderem S. 29-50, 64-80.

8 Nicht ganz geklärt ist, wann Grimmelshausen tatsächlich mit dem Verfassen seiner Kriegsromane begonnen hat. Im auf das Jahr 1668 datierten „Beschluss“ der „Contiuatio“ zum Simplicissimus Teutsch behauptet er, er habe den Roman ,in seiner Jugend zum theil geschrieben / als er noch ein Mußquetirer gewesen“. Grimmelshausen: Simplicissimus Teutsch (Anm. 4), S. 699. Die wichtigste Schreibphase lässt sich aber wohl auf die Mitte der 1660er Jahre datieren, als Grimmelshausen die Wirtschaft zum Silbernen Stern in Gaisbach führte. Vgl. hierzu als Überblick Heiner Boehncke/Hans Sarkowicz: Grimmelshausen. Leben und Schreiben. Vom Musketier zum Weltautor. Frankfurt/M.: Greno 2011, vor allem S. 334-361. 
Doch zuerst ein paar einleitende Worte zur Struktur des Bändchens: Es handelt sich hierbei um ein dialogisch aufgebautes Moraltraktat, in dem, wie in der „Vorred“ auch erläutert wird, diverse Themen, wie etwa die Dichtkunst, die Philosophie, der Tanz, die Schönheit, der Wein oder die Frauen, in einem als „Satz“ und „Gegensatz“ voneinander getrennten, forcierten Pro und Contra präsentiert werden. Daraufhin folgt jeweils ein kurzes Fazit, in dem zumeist eine relativierende Position mit stark moralisierendem Einschlag eingenommen wird. Bei alldem präsentiert sich Grimmelshausen unter dem Pseudonym Samuel Greifnson vom Hirschfeld zumeist als ein insgesamt wenig revolutionärer, eher konservativer Geist mit einer deutlichen Neigung zu sogenannten altdeutschen Positionen.

Diese Einschätzung gilt allerdings nur zum Teil. Vor allem die erste und die letzte Gegenüberstellung in der „Vorred“ und dann im „Zehende[n] Satz / vom Krieg“, die hier im Folgenden im Mittelpunkt stehen sollen, gewinnen bei einem genaueren Hinschauen eine ganz eigene Dynamik. Und sie sind es auch, die sowohl die angekündigte grundsätzliche Selbsteinführung von Grimmelshausen als Autor als auch eine sehr spezifische Ankündigung und Positionierung des zwei Jahre später auf den Buchmarkt folgenden Simplicissimus Teutsch enthalten.

Zunächst zur „Vorred“: Grimmelshausen führt sich in ihr gewissermaßen mit einer Autor(selbst)beschimpfung in die literarische Welt ein. Ein fiktiver Kritiker namens Momus tritt auf und wirft dem Verfasser des vorliegenden Bandes dessen mangelnde Bildung vor. Er sei eben nur ein Musketier, der dem Krieg entlaufen sei und nun mit ein paar aufgeschnappten Wissensbrocken renommiere:

Dann lieber was wolten doch vor Nutzbarkeit und Lehren von einem solchen Kerl wie der Author ist / zu hoffen seyn? Man weiß ja wohl daß Er selbst nichts studirt, gelernet noch erfahren: sondern so bald er kaum das $\mathrm{ABC}$ begriffen hatt / in Krieg kommen / im zehenjährigen Alter ein rotziger Musquedirer worden [...]; Zwar vermercket man in seinem Stylo wohl / was Er weiß und vermag; In deme er nicht recht orthographicè schreiben kan; So ist auch kein Ordnung: viel weniger eine Liebligkeit in seinem gantzen Buch zu finden; In Summa es mangelt überall ahn Saltz und Schmaltz / nichts ist verhanden als ein wercklichs Mischmasch / von lauter Fähl und Mängeln zusammen gestickelt; [...] So gehets aber / wann Musquetirer die Feder brauchen und ungelehrte Bücher schreiben wollen [...]. ${ }^{9}$

Insgesamt steht für den Kritiker fest, dass der Verfasser besser daran getan hätte, bei seinem Kriegshandwerk zu verbleiben, als die Welt mit einem solchen Machwerk zu belästigen.

9 Grimmelshausen: Satyrischer Pilgram (Anm. 7), S. 6. Zitate daraus werden im Text mit der Sigle SP und Seitenangabe nachgewiesen. 
Der „Author“ selbst meldet sich in der „Gegenschrifft“ (SP, S. 8-12) zu Wort und verteidigt sich gegen die Vorwürfe. Für sein Schicksal eines in den Krieg Geworfenen könne er schließlich nichts:

Betreffend meiner Person Wenigkeit / daß ich nehmlich nichts studirt, sonder im Krieg uffgewachsen / und allda wie ein anderer grober Esel keine Wissenschafften gefast habe / [...] solches ist niemand leider als mir; Aber da wisse du und deinige / daß ich mich vor keinen Doctorem außgebe / [...] daß dennoch mit dem wenigen so ich erfahren / meinem Nebenmenschen zu dienen begehre [...]. (SP, S. 9)

Überhaupt wende er sich gar nicht an seinen Kritiker, sondern schreibe „nur vor meines gleichen einfältige Leut“" (SP, S. 9).

Interessant ist hier die Sprachgebung. Eingesetzt wird in der „Gegenschrifft“ mit einem „MOme“ (SP, S. 8) - also einer Ansprache des Gegenübers im korrekten lateinischen Vokativ, und auch im obigen Zitat wird „Doctorem“ im korrekten lateinischen Akkusativ gesetzt. Irgendetwas scheint an Bildung also doch vorhanden zu sein, und auch in der Folge werden im Text immer wieder durchaus umfangreiche gelehrte Wissensbestände aktiviert. In einem scharfen Kontrast dazu stehen wüste Beschimpfungen und ein gelegentlich durchbrechender vulgärer Grundton, mit dem die Herkunft des Autors direkt aus dem Heerlager markiert wird. Gegen die Kritik am verwirrend-widersprüchlichen Buchtitel des Satyrischen Pilgram wendet er etwa ein: „Du darffsts dich des Tituls halber auch nicht sonderlich bekümmern / dann ichs nicht uff diese Art zu machen begehrt / da der wohl uffgeputzte Kopf einer gemeinen Huren den besch: Hindern verkauffen soll“ (SP, S. 10).

Der Verfasser positioniert sich hier also erkennbar und explizit nicht nur außerhalb des gelehrten, sondern auch des gepflegten Diskurses. Indem in der „Vorred“ Konzessionen und Attacken einander abwechseln, bleibt er hier insgesamt allerdings noch etwas unentschieden.

\section{Gelehrtes Wissen und persönliche Erfahrung - „Zehender Satz / vom Krieg“}

Diese zunächst noch eher trotzig-defensive Haltung verschiebt sich dann im finalen und sicherlich auch wichtigsten Stichwort des Satyrischen Pilgram nochmals merklich. Das Phänomen des Krieges, um das es hier geht, wird im ersten Teil, der die für ihn sprechenden Argumente versammelt, geradezu schulmäßig mit Rückgriff auf Platon, Aristoteles sowie zahlreiche weitere Autoritäten und historische Beispiele durchgenommen. Mit aller nun offenbar doch vorhandenen 
Gelehrsamkeit und unter einem Gewitter illustrer Namen von der Antike bis zur Jetztzeit des zu Ende gegangenen Dreißigjährigen Krieges werden dabei die üblichen Topoi des Kriegslobs abgeschritten.

Im darauffolgenden „Gegensatz“ reicht dann allerdings eine einzige Feststellung, um dieses zuvor kunstvoll aufgebaute Kartenhaus wiederum zum Einsturz zu bringen: „Es scheinet als wan dieser Discurs allerdinges keines Gegensatzes bedörffe / dieweil im verwichenen Teutschen Kriege ein ieder genugsam / und zwar mit unwiederbringlichem Schaden / erfahren haben wird / was der Krieg sey?“ (SP, S. 156)

Die zuvor vorgebrachte Argumentation für den Krieg ist also angesichts des gemeinsam Erlebten unmittelbar als ein sich selbst entlarvender Unsinn erkennbar - sie ist reines Wortgeklingel. Zur Gewähr dient dabei ein Maximalkollektiv von Zeitgenossen, das über die entsprechende konkrete historische Erfahrung verfüge: „ein ieder“. Grimmelshausen spielt hier also ganz offensiv das selbst Erlebte gegen das Buchwissen aus und sucht dabei den Schulterschluss mit seiner Leserschaft.

Allerdings ist nun genau diese Einigkeit des Wissens auch schon wieder in Gefahr: „Dieweiln aber seit dem Friedenschluss so ein Hauffen junger Schnautzhahnen uffgewachsen / die nur deßwegen gern einen Krieg sehen / weil sie nit wissen was Krieg ist / als mueß ich ihnen ein wenig etwas zum besten thuen“ (SP, S. 156).

Das gemeinsame historische Erleben, das zuvor aufgerufen worden ist, ist also gerade jetzt, rund 20 Jahre nach Kriegsende, im Begriff wieder verloren zu gehen. Deshalb soll nun die als essenziell begriffene Erfahrung, die damit verbunden ist, textuell restituiert werden. Mit anderen Worten: Der durch die Generationenfolge begrenzte Raum der temporalen Gegenwart als dem kollektiven Bewusstsein der gleichzeitig lebenden Menschen soll ,gedehnt' werden, indem eine langsam aus ihr herausrutschende gemeinsame Erfahrung diskursiv aktualisiert und dadurch erneuert wird.

Tragend scheint hier ein Generationenkonzept, das an die modernen sozialen Fassungen dieses Begriffs zumindest in einigen Hinsichten anschließbar ist. ${ }^{10}$

10 Vgl. dazu die instruktiven Einträge von Hans-Joachim von Kondratowitz: Generationen Generationentransfer. In: Friedrich Jäger (Hg.): Enzyklopädie der Neuzeit. Stuttgart: Metzler 2005-2012. Bd. 4. Stuttgart: Metzler 2006, Sp. 443-456. Vgl. grundlegend zum sozialen Generationenbegriff der Moderne Karl Mannheim: Das Problem der Generationen (1928). In: K.M.: Wissenssoziologie. Auswahl aus dem Werk. Hg. von Kurt H. Wolff. Neuwied/Berlin: Luchterhand ${ }^{2} 1970$, S. 509-565. Vgl. allgemeiner auch Sigrid Weigel: Genea-Logik. Generation, Tradition und Evolution zwischen Kultur- und Naturwissenschaften. München: Fink 2006. 
Nach der klassischen Definition von Wilhelm Dilthey aus dem Jahr 1875 handelt es sich hierbei um

einen engeren Kreis von Individuen, welche durch Abhängigkeit von denselben großen Tatsachen und Veränderungen, wie sie in dem Zeitalter ihrer Empfänglichkeit auftraten, trotz der Verschiedenheit hinzutretender anderer Faktoren zu einem homogenen Ganzen verbunden sind. ${ }^{11}$

Der Dreißigjährige Krieg gehört zweifellos zu den hier ins Spiel gebrachten „großen Tatsachen“, die diejenigen, die ihn erlebt haben, generationell von den anderen unterscheiden, bei denen dies nicht mehr der Fall ist. Sie wissen, was Krieg bedeutet und warum er abzulehnen ist.

Doch liegen auch Differenzen zum Generationenkonzept vor, wie es dann später ausformuliert werden sollte. Dieses orientiert sich seit dem späten 18. Jahrhundert vor allem an der Gruppe der Jüngeren, die, wie es ja auch bei Dilthey heißt, in ihren besonders empfänglichen Jahren bestimmten prägenden Erfahrungen ausgesetzt gewesen seien, was in der Folge wiederum zu entsprechenden Mentalitätsänderungen geführt habe. Sie passen sich der jeweils neuen Zeit an und gestalten sie mit, während die Alten zurückbleiben.

Im hier vorliegenden Fall ist es nun aber so, dass die entscheidenden Erfahrungen erst einmal allen lebenden Menschen zugeschrieben werden, was natürlich auch auf die schiere Länge des zugrundeliegenden Geschehens zurückgeführt werden kann. Knapp ein halbes Jahrhundert nach dem Beginn des Dreißigjährigen Krieges lebt praktisch niemand mehr, dessen Persönlichkeitsentwicklung schon zuvor soweit abgeschlossen gewesen wäre, dass er sich hiervon nicht mehr hätte beeindrucken lassen.

Zugleich wird nun ausgerechnet der Gruppe der Jungen - den „Schnautzhahnen“ - attestiert, dass ihnen genau diese Dimension des Erlebnisses fehle, weswegen der Krieg ihnen nicht mehr gegenwärtig sei und damit als etwas unbedingt zu Vermeidendes erscheine. Sie seien für die Sirenengesänge von Ruhm, Macht und der Chance auf gesellschaftlichen Aufstieg, die den ersten Teil der Darstellung bestimmten, fatalerweise bereits wieder empfänglich. ${ }^{12}$

11 Wilhelm Dilthey: Über das Studium der Geschichte der Wissenschaften vom Mensch, der Gesellschaft und dem Staat (1875). In: W.D.: Gesammelte Schriften. Bd. V: Die Geistige Welt. Einleitung in die Philosophie des Lebens. Erste Hälfte: Abhandlungen zur Grundlegung der Geisteswissenschaften. Stuttgart/Göttingen: Teubner/Vandenhoeck \& Ruprecht 1990, S. 37.

12 Eine solche Figur taucht im zehnten und letzten Band des simplicianischen Zyklus mit dem namenlosen Ich-Erzähler des Wunderbarlichen Vogel-Nests dann auch selbst auf. Vgl. Hans Jacob Christoffel von Grimmelshausen: Das wunderbarliche Vogel-Nest. Zweiter Teil. In: H.J.C.G.: Werke in drei Bänden. Hg. von Dieter Breuer. Frankfurt/M.: Deutscher Klassiker Verlag 1989ff. Bd I.2. 
Der Krieg stellt für Grimmelshausen also offenbar eine sehr umfangreich bemessene Gegenwart dar, die das Bewusstsein gleich mehrerer Generationen tiefgreifend $\mathrm{zu}$ prägen vermocht hatte. Darüber $\mathrm{zu}$ sprechen, war - wie bereits erwähnt - solange überflüssig, wie es in dieser Frage einen unhinterfragten Konsens gab, dessen drohenden Verlust er nun aber diagnostiziert. In welchem Maße er damit sachlich Recht hatte oder auch nicht, scheint mir dabei weniger zentral zu sein als sein daraufhin gefasster Plan, dieser von ihm wahrgenommenen Änderung der Mentalität mit einem Handlungs- und Schreibprogramm energisch entgegenzutreten.

\section{Vom Festhalten der Erfahrung des Krieges in der Gegenwart}

Es geht Grimmelshausen also darum, das Gedenken an den Krieg festzuhalten und die Erfahrung gleichsam auf Dauer zu stellen. Derartige Formen der memoria waren als Ziel von Kunstwerken in der Frühen Neuzeit und auch schon in früheren Zeiten natürlich keinesfalls ungewöhnlich. Gleichwohl liegt hier auch in dieser Hinsicht ein Sonderfall vor, denn üblicherweise waren es positive Dinge, deren Andenken aufrechterhalten werden sollte: große Siege, heroische Taten oder göttliche Rettung. Das Gegenteil schien der memoria gemeinhin eher nicht würdig. In der Antike etwa konnten missliebige Verstorbene gar mit einer damnatio memoriae, also einer aktiv betriebenen Tilgung des Andenkens bedacht werden, wobei im Vergessenwerden die eigentliche Strafe liegen sollte. Und auch die zahlreichen Pestsäulen der Frühen Neuzeit erinnerten nicht eigentlich an das Wüten der Seuche selbst, sondern wurden vielmehr zum Dank für ihr jeweiliges Erlöschen aufgestellt. Vor diesem Hintergrund erscheint es als ein zumindest nicht ganz üblicher Schritt, gerade das Negative des Erlebten konservieren zu wollen. ${ }^{13}$ Die Motivation ist gleichwohl mehr als klar: Da die Menschen offenbar

Frankfurt/M.: Deutscher Klassiker Verlag 1992, S. 449-650. Dieser Roman spielt in der Jetztzeit des Schreibens, also rund ein Vierteljahrhundert nach dem Ende des Dreißigjährigen Krieges, dessen Folgen hier nur noch von Ferne zu spüren sind und dessen Gegenwart sich tatsächlich weitgehend verloren $z u$ haben scheint. Der Ich-Erzähler, der kein wirklicher Zeitgenosse des großen Krieges mehr ist, meldet sich gegen Ende des Buches freiwillig in den französisch-niederländischen Krieg der Jahre 1672 bis 1678, in dem er dann gleichsam im Schnelldurchlauf (vor allem Kapitel XXIII, S. 617-624) die entsprechenden Negativerfahrungen gesammelt und geballt nachholt.

13 Eine Kultur einer derartigen memoria des Negativen hat sich wohl erst im 20. Jahrhundert vor allem in Deutschland im Umgang mit der eigenen NS-Vergangenheit etabliert. Allerdings hat 
intuitiv zum Krieg neigen, wenn ihnen nicht seine schlimmsten Seiten bewusst sind, muss man diese denjenigen, die ihn nicht am eigenen Leibe erfahren haben, umso eindringlicher vor Augen führen.

Grimmelshausen setzt in der Folge gleich damit an, das Wesen des Krieges zu erläutern. Dies geschieht zunächst, ohne dass konkrete historische Momente genannt würden, in einem verallgemeinernden Präsens. Verwendet wird bei alldem ein bewusst volkstümlicher Stil, der weitgehend ohne gelehrte Elemente auskommt. Recht eindrücklich ist hier etwa die Reihe von Tiervergleichen, mit denen die Entmenschlichung des Soldaten im Krieg illustriert wird: An solchen Stellen wendet sich der Autor nun wirklich an ,seinesgleichen' - das heißt an ein formal ungebildetes Volk:

und so bald er immer das entsetzlichste Lermen-geschrey erhöret / so mueß er schon resolvirt seyn / entweder selbst zu sterben / oder seinen Neben-Christen-Menschen umzubringen wie ein Viehe; Bald mueß er sich mit grosser Arbeith und Gefahr entweder in die Erden graben wie ein Dachs; oder in ein Morastig Wasser begeben wie ein Frosch; oder uff ein Mahl oder Maur klimen wie ein Marder / weil er des Dedali Flügel nicht hat wie ein Vogel / und bald mueß er mitten im Plitz und Feuer schweben wie ein Salamandra, [...]; Jetzt muß er schleichen wie ein Schneck ietzt nachjagen wie ein Hund / bald mueß er fliehen wie ein Haaß; und ehe er sichs versiehet / so mueß er still liegen wie ein Bäer / der seine Tapen sauget [...]. (SP, S. 157)

An einer Stelle der Argumentation wird dieses Präsens allerdings doch einmal durchbrochen. Etwa auf der Mitte der Strecke erinnert der Sprecher daran, dass seine als zeitlos auftretende Analyse des Krieges sehr wohl auf einem sehr zeitgebundenen Erlebnis beruht: „Ohne Ruhm zu melden / ich bin ehemalen auch darbey gewesen / da man einander das weisse in den Augen beschaute / kan derowegen wohl Zeugnüß geben“ (SP, S. 158).

Der Autor stellt sich also als persönlicher Gewährsmann hinter seinen Text. Dies hat bekanntlich durchaus ein Fundament in der Biografie Grimmelshausens, der selbst in jungen Jahren nach einer Plünderung seines Heimatortes Gelnhausen unter die Soldaten gefallen war und weite Teile des Krieges am eigenen Leibe mitgemacht hat. Gleichwohl wird im Satyrischen Pilgram mit diesem biografischen Hintergrund auch gespielt. Wenn der Kritiker in der „Vorred“ unwidersprochen behauptet, dass der Autor, „so bald er kaum das ABC begriffen hat / in Krieg kommen / im zehenjährigen Alter ein rotziger Musquedirer worden“ (SP, S. 6), so stimmt dies nur zum Teil mit den historischen Fakten überein, denn Grimmelshausen selbst war damals wohl doch schon zwölf oder dreizehn Jahre

auch diese ihre Grenzen. Der zentrale Holocaustgedenktag etwa wurde mit dem 27. Januar auf das Datum der Befreiung des Konzentrationslagers Auschwitz im Jahr 1945 gelegt. 
alt und hatte somit nach dem Elementarunterricht wahrscheinlich bereits zwei oder drei Jahre lang die Lateinschule besucht. Mit Verschiebungen und Stilisierungen ist auch hier also stets zu rechnen. An der persönlichen Teilnahme an Kampfhandlungen, die an dieser Stelle der Darstellung Autorität verleihen soll, ist aber wohl nicht zu zweifeln.

Bei alldem steht die Berufung auf die eigene Erfahrung hier am Ende aber doch recht unverbunden neben der Reihe der allgemeinen Aussagen über das Wesen des Krieges. Die offenbar empfundene Notwendigkeit, mitten in der Analyse nochmals an ihr Fundament in der Sache zu erinnern, zeigt zugleich die Schwäche der Argumentation auf. In gewisser Weise haben sich die Ausführungen von ihrem gelehrten Gegenstück, das ja ebenfalls auf abstrakte Allgemeinheit zielt, strukturell nicht gelöst und können es im gegebenen Rahmen wohl auch gar nicht.

\section{Das erhoffte Remedium - der Übergang vom Traktat zum Roman}

Genau dieser Mangel wird im kurzen „Nachklang“ nochmals aufgegriffen:

Ich gestehe gern / daß ich den hundersten Theil nicht erzehlet / was Krieg vor ein erschreckliches und grausames Monstrum seye / dann solches erfordert mehr als ein gantz Buch Papier / so aber in diesem kurtzen Wercklein nicht wohl einzubringen wäre / Mein Simplicissimus wird dem günstigen Leser mit einer andern und zwar lustigern Manier viel Particularitäten von ihm erzehlen / indessen halte ich darvor / es sey uns Christen nichts ohnanständiger als der Krieg / den wir wieder einander führen [...]. (SP, S. 160) $)^{14}$

Geradezu programmatisch wird hier ein Übergang von der notwendig defizitären summarischen und verallgemeinernden Darstellung der Kriegsgräuel im Satyrischen Pilgram zur Schilderung der „Particularitäten“ im Simplicissimus Teutsch geschaffen. An die Stelle des abstrahierenden Räsonierens soll im konkreten Nachvollzug der Erlebnisse eines einzelnen Individuums das vergegenwärtigende Erzählen treten. Dass auch dieses Erzählen einer einzelnen Figur nicht wirklich

14 Es sei hier nicht verschwiegen, dass der Rest des „Nachklangs“ dann doch in eine andere Richtung weist und auf seine Art ebenfalls eine Mittelposition zwischen ,Satz‘ und ,Gegensatz' einnimmt. Auch wenn die strikte Ablehnung jedes Krieges unter den Christen weiterhin gültig bleibt, wird zugleich zur Einigkeit im als notwendig gesehenen Kampf gegen das expandierende Osmanische Reich aufgerufen. Diesem historischen Großkonflikt steht sein Höhe- und Wendepunkt mit der Belagerung Wiens durch die Türken im Jahr 1683 noch bevor. 
ausreichen wird, den Krieg insgesamt zu fassen, sondern dafür, wie es ausdrücklich heißt, „mehr als ein gantz Buch Papier“ nötig sein wird, deutet sich hier ebenfalls bereits an. ${ }^{15}$ Es ist an dieser Stelle schon zu erahnen, dass sich der Simplicissimus zu einer ganzen Reihe von Büchern mit diversen Sprosserzählungen verschiedener Figuren fortentwickeln wird.

Diese zuerst im Satyrischen Pilgram geforderte Überwindung des eher ins Allgemeine gehenden Sprechens über den Krieg zugunsten der Vergegenwärtigung durch individuelle „Particularitäten“ findet in den eigentlichen simplicianischen Schriften Grimmelshausens allerdings nicht nur in der Erzählpraxis, sondern auch in romaninternen Reflexionen nochmals eine eindrucksvolle Bestätigung. Im Seltzamen Springinsfeld, dem zweiten Sprossroman des Simplicissimus Teutsch, soll die Titelfigur in einer romaninternen Erzählsituation gegenüber einer Gruppe von Zuhörern, unter denen sich auch der alte Simplicissimus selbst befindet, von seinen Erfahrungen berichten. Von außen scheint er dazu geradezu paradigmatisch geeignet, hat doch der Krieg diesem Invaliden durch den Verlust des rechten Beines seine Wirkung direkt und unauslöschlich in den Körper eingeschrieben. ${ }^{16}$ Springinsfeld schafft es allerdings kaum, wirklich bei dem von ihm Erlebten zu bleiben. Gleich mehrfach geht er über $\mathrm{zu}$ allgemeinen Reflexionen über Unterschiede zwischen den verschiedenen Kriegen, an denen er teilgenommen hat, oder auch zu allgemeinen Definitionen von Krieg. ${ }^{17}$ Der zuhörende Simplicissimus schreitet hier mit einem Machtwort ein: „Simp: fiele ihm in die Rede und sagte / entweder redestu im Schlaf oder wilst wieder aus dem Weg tretten / du wilst den Krieg underscheiden und vergist abermal deiner eignen Person / sage darvor wie es dir selbst gangen?“18

15 Indirekt annonciert wurde dies auch schon in den Attacken des Kritikers in der „Vorred“: „Zu deme hat der Phantast ein Werck vor / daß sich ad infinitum hinein erstreckt / welches wohl jemand anderer als er ist / wann er schon Mathusalems Alter erreichte / nicht enden und hinauß führen könte“ (SP, S. 7).

16 Vgl. zur Geschichte der Kriegsinvaliden, deren Versorgung und Schicksal in den Söldnerheeren des 17. Jahrhunderts besonders trist und deren Ruf entsprechend schlecht war, Achim Hölter: Die Invaliden. Die vergessene Geschichte der Kriegskrüppel in der europäischen Literatur bis zum 19. Jahrhundert. Stuttgart/Weimar: Metzler 1995, speziell zur Figur des Springinsfeld vgl. S. 70-75.

17 U.a. Battafarano betont, dass Springinsfeld gar nicht prinzipiell gegen den Krieg ist. Insgesamt hält er an dem im gesamten Zyklus als irrig vorgeführten Glauben fest, dass man den Krieg für seine Zwecke nützen könnte, wenn man es nur ausreichend geschickt anstellte. Vgl. Battafarano: Simpliciana Bellica (Anm. 1), S. 96 f.

18 Hans Jacob Christoffel von Grimmelshausen: Der seltzame Springinsfeld. In: H.J.C.G.: Werke in drei Bänden. Hg. von Dieter Breuer. Frankfurt/M. 1989ff. Band I.2. Hg. von Dieter Breuer. Frankfurt/M.: Deutscher Klassiker Verlag 1992, S. 153-295, hier S. 223. 
In der konsequenten Fortführung der Ankündigung aus dem Satyrischen Pilgram wird hier nochmals das Konkrete der spezifischen, selbst erlebten historischen Situation eingefordert, das auch im Abstand von über 20 Jahren letztlich wichtiger ist als jegliche Gemeinaussage über den Krieg.

Auch hier bleibt also das im Satyrischen Pilgram zuerst entwickelte Programm noch gültig. Es geht darum, mit einem Erzählen von individuellen Kriegserlebnissen diesen als Erfahrungshintergrund fest- und gegenwärtig zu halten, damit eine Wiederholung seiner Schrecken weiterhin vermieden werden kann. Notwendig erscheint dies in dem historischen Moment, in dem die zugrundeliegende kollektive Erfahrung langsam verloren zu gehen droht. Natürlich handelt es sich dabei noch nicht um emphatische Gegenwartsentwürfe oder den Wandel affirmierende Zukunftsvisionen, die das Geschichtsdenken später prägen werden. Gleichwohl scheint hier bereits ein komplexes Verständnis der historischen Zeit unter Einbezug der Kategorie der Gegenwart auf. Die Rolle der Vergangenheit ist dabei eine doppelte: Während die ältere Vergangenheit des Krieges ein Negativexempel darstellt, wird die jüngere Vergangenheit der Nachkriegszeit, die in die Gegenwart mündet, als eine sich selbst verändernde verstanden, in der eine durch Erfahrung hervorgerufene positive Erkenntnis nach dem Ablauf einer Generation wieder zu schwinden beginnt. In der eigentlichen Gegenwart selbst wiederum soll eine Maßnahme genau hiergegen ergriffen werden. Diese stellt allerdings keinen einfachen Rückgriff auf ein Früheres dar, sondern besteht vielmehr in dessen medialer Transformation. Das Erlebte soll zu einer Erzählung werden, gerade damit die Dinge in der Realität nicht wiederkehren. All dies geschieht schließlich um der Sicherung einer weiteren Zukunft der Menschen willen. 